\title{
Resultado funcional do tratamento artroscópico da instabilidade do ombro sem a lesão do lábio da glenóide*
}

\author{
Functional results of arthroscopic treatment of \\ shoulder instability without glenoid labral lesion
}

\author{
WALDO LiNo JÚNIOR ${ }^{1}$, WILLIAM Dias BELANGERO²
}

\section{RESUMO}

Objetivo: Tratar a instabilidade anterior e ântero-inferior do ombro sem lesão do lábio da glenóide por via artroscópica por meio de capsulorrafia térmica e fechamento do espaço rotador e avaliar os resultados quanto à estabilidade è̀ melhora funcional. Métodos: Entre janeiro de 1998 e junho de 2002, 11 pacientes foram submetidos a esse procedimento, imobilizados por cinco semanas e após tratados por fisioterapia. Todos os pacientes foram avaliados no pré e pós-operatório pelas escalas de Neer, Rower e UCLA e, no pós-operatório, pela escala de ASES. Resultados: Durante o período de seguimento entre 46 e 97 meses - média de 75,54 meses - todos os ombros permaneceram estáveis. Comparando-se a evolução do pré ao pós-operatório, houve melhora de acordo com a escala de Neer, na qual nenhum paciente apresentou resultado satisfatório no pré-operatório e oito tiveram resultado satisfatório no pós-operatório. Segundo a escala de Rowe, a média préoperatória foi de 36,8 e, no pós-operatório, de 81,6 pontos. Conforme a escala da UCLA, a média no pré-operatório foi de 23,45 e, no pós-operatório, de 31,18 pontos. De acordo com todas essas escalas houve melhora estatisticamente significativa $(p=0,05)$. A escala da ASES mostrou média

* Trabalho realizado na Real e Benemérita Associação Portuguesa de Beneficência de São Paulo - Hospital São Joaquim - São Paulo (SP), Brasil.

1. Mestre, Chefe de Equipe Ortopédica da Real e Benemérita Associação Portuguesa de Beneficência de São Paulo - Hospital São Joaquim - São Paulo (SP), Brasil.

2. Doutor, Professor do Departamento de Ortopedia e Traumatologia da Faculdade de Ciências Médicas da Universidade de Campinas - UNICAMP Campinas (SP), Brasil.

Endereço para correspondência: Alameda Santos, 211, cj. 209 - 01419-002 São Paulo (SP) - Brasil. Tel.: (11) 3289-1404.

Recebido em 14/6/05. Aprovado para publicação em 27/7/07.

Copyright RBO2007 pós-operatória de 77,2 pontos. Conclusão: O procedimento artroscópico analisado confere estabilidade e melhora funcional.

Descritores - Instabilidade articular/cirurgia; Luxação do ombro; Artroscopia/métodos; Resultado de tratamento

\section{ABSTRACT}

Objective: Anterior and anterior-lower instability of the shoulder without a glenoid labral lesion was arthroscopically treated by thermal capsulorrhaphy and closing of the rotator space, and results were evaluated for stability and functional improvement. Methods: From January 1998 to June 2002, 11 patients were submitted to this procedure, immobilized for five weeks, and then treated by physiotherapy. All patients were evaluated pre- and post-operatively by the Neer, Rower, and UCLA scale, and in the post-operative period, by the ASES scale. Results: During the follow-up period-between 46 and 97 months, mean 75.54 months - all shoulders were stable. Comparing the evolution from the pre-to the post-operative period, there was an improvement according to the Neer scale, and no patient displayed satisfactory results in the preoperative period and eight had satisfactory results in the post-operative period. According to the Rowe scale, the preoperative mean was 36.8, and the post-operative mean was 81.6 points. The UCLA scale presented a pre-operative mean of 23.45, and a post-operative mean of 31.18 point. According to all scales, there was a statistically significant improvement $(p=0.05)$. The ASES scale showed a postoperative mean of 77.2 points. Conclusion: The arthroscopic procedure being studied provides stability and functional improvement.

Keywords - Joint instability/surgery; Shoulder dislocation; Arthroscopy/methods; Treatment outcome 


\section{INTRODUÇÃO}

Consideramos que a redução do volume capsular glenoumeral associada ao fechamento do espaço rotador via artroscópica é tratamento eficaz na instabilidade anterior e ânteroinferior do ombro que apresenta o lábio da glenóide íntegro.

Essa lesão é observada na instabilidade multidirecional do ombro, onde existe distensão do complexo capsuloligamen$\operatorname{tar}^{(1)}$, e apresenta evolução contínua e progressiva ${ }^{(2)}$. Em certos casos, a ação de microtraumas e estresses repetitivos podem levar a instabilidade a uma direção preferencialmente anterior ou ântero-inferior ${ }^{(3)}$.

Esses casos são tratados inicialmente por exercícios de reforço muscular e de propriocepção; a maioria evolui com resultados favoráveis. Alguns ombros, entretanto, permaneceram instáveis e devem ser submetidos ao tratamento cirúrgico. Reavaliamos os pacientes a quem submetemos à capsulotomia térmica e fechamento do espaço rotado, objetivando analisar os resultados funcionais proporcionados pelo procedimento, além de suas eventuais complicações.

\section{MÉTODOS}

Entre janeiro de 1988 e junho de 2002, 11 pacientes foram submetidos à redução do volume capsular térmico (capsulorrafia térmica) e fechamento do espaço rotador no Hospital São Joaquim (São Paulo).

Critérios de inclusão - Foram incluídos neste estudo os pacientes com instabilidade multidirecional do ombro, predominantemente anterior ou ântero-inferior, com no mínimo quatro episódios de luxação, sendo todos involuntários. Todos deveriam ter sido submetidos a tratamento por fisioterapia por no mínimo seis meses. No exame artroscópico, o lábio e a borda da glenóide deveriam estar íntegros e sem desinserção capsular aparente.

Técnica cirúrgica-Os procedimentos foram realizados por via artroscópica, em decúbito lateral com tração cutânea e sob anestesia geral. Foram usados os portais anterior e posterior. Inicialmente foi realizado um exame articular para a cápsula, o lábio da glenóide e a superfície articular glenoumeral. A capsulorrafia era realizada utilizando o sistema térmico de Holmio Laser ${ }^{\circledR}$ (Ho:YAG Trimedyne Inc. 2801 Barranca, Irvine, CA 92714), ajustado com potência de $27 \mathrm{~W}, 55 \mathrm{~Hz}$ e $0,5 \mathrm{~J}$. Com o controle visual artroscópico no portal anterior e a ponteira do sistema posicionada no portal posterior, o laser era aplicado na cápsula inferior e por toda a extensão do ligamento glenoumeral inferior, observando-se a redução do volume no recesso axilar. Deslocando o artroscópio para o portal pos- terior, o laser era aplicado no restante dos ligamentos inferior e médio. $\mathrm{O}$ padrão de aplicação era gradeado ou por faixas interrompidas por tecido não tratado, conservando uma superfície vascularizada. O controle da redução do volume capsular foi feito pela observação visual e pela mudança da coloração do tecido tratado, que passava de branco para amarelado.

Após o tratamento capsular, era feito o fechamento do espaço rotador pela aproximação da borda superior do tendão do músculo subescapular e a anterior do tendão do músculo subespinhoso, que eram fixadas com fios Ethibond $^{\circledR} \mathrm{n}^{\mathrm{0}} 5$. Esses pontos eram posicionados da porção mais lateral para a mais medial do tendão do subescapular em direção à borda anterior da glenóide. A sutura era feita pela passagem de uma agulha de peridural pelo portal anterior através da borda anterior do tendão do supra-espinhoso, por onde era avançado um fio Prolene ${ }^{\circledR}$ no 2. A agulha era então retirada pelo mesmo portal anterior. Um sistema de apreensão de fio (suture grasper) recuperava o fio que era exteriorizado. O fio Prolen $e^{\circledR} n^{\circ}$ 2 era substituído pelo fio Ethibond ${ }^{\circledR}$ nํㅜ 5, que era, então, fixado por nós. O número de pontos variou de três a seis, com media de 4,4. Consideramos insuficiente o fechamento do espaço apenas pela aproximação do ligamento glenoumeral superior ao médio. Os pacientes eram imobilizados com tipóia funcional por cinco semanas, sendo estimulada a mobilização da mão e do cotovelo. A reabilitação fisioterápica era iniciada na quarta semana, com mobilização passiva assistida nas duas primeiras semanas seguida por exercícios isométricos com theraband e exercícios proprioceptivos até a recuperação da força e mobilidade.

Critérios de avaliação - Os pacientes foram avaliados no pré e pós-operatórios pelas escalas de Neer, como descrito por Ellman et $a l^{(4)}$, Rowe et $a l^{(5)}$, e UCLA, como descrito por King et $a l^{(6)}$. No pós-operatório foram avaliados pela escala de ASES, como descrito por Green et al $l^{(7)}$.

\section{RESULTADOS}

Entre janeiro de 1998 e julho de 2002, 11 pacientes foram operados pela técnica acima descrita. O seguimento pós-operatório médio foi de 75,54 meses (46-97). Entre os pacientes, oito eram do sexo masculino, com média de idade de 28 anos (14-56); em seis pacientes a lesão era do lado esquerdo e, em cinco, do lado direito. O lado dominante estava acometido em cinco pacientes. Apenas um paciente apresentava luxação bilateral e apenas o lado esquerdo foi tratado cirurgicamente. A média de idade do primeiro episódio de luxação foi de 24,5 anos. O número máximo de luxações foi de 40 episódios, neste estudo, tendo número de luxações mediana de 20 episódios 
(4-40). Todos os pacientes referiram a percepção da ocorrência de subluxação articular.

Entre os pacientes, oito $(72,7 \%)$ queixavam-se de dor. Ao exame físico, todos os pacientes apresentavam sinal de apreensão com o braço a $90^{\circ}$ de abdução e rotação externa e teste da gaveta positivo. A amplitude do movimento ativo no pré-operatório foi considerada, observando-se média de flexão de $180^{\circ}$, abdução de $175^{\circ}$, rotação interna em T9 e rotação externa de $130^{\circ}$. Ao exame artroscópico, dois pacientes apresentavam variação anatômica, em que o ligamento glenoumeral inferior apresentava forma de cordão inserido próximo ao ligamento glenoumeral superior e ausência do lábio anterior (complexo de Buford). A recuperação da amplitude de movimento ocorreu de forma gradativa. A flexão máxima foi alcançada em média no sexto mês (três a 14 meses). Houve redução na rotação externa passiva: média de $80^{\circ}$. A duração média do tratamento fisioterápico foi de 36,6 sessões (20-50).

Durante este estudo não foi constatada nenhuma recidiva da luxação em ombros operados. Não foi feita nenhuma reavaliação artroscópica, o que não nos permite afirmar se houve algum caso de extensa necrose capsular; contudo, a ausência de recidiva sugere a integridade capsular. Não observamos nenhum caso de capsulite adesiva e não detectamos sinais radiológicos de artrose articular.

Análise da escala de Neer - Segundo a classificação de Neer, 11 pacientes apresentavam resultado insatisfatório no pré-operatório. No pós-operatório, oito pacientes mostraram resultados satisfatórios e três pacientes, resultados insatisfatórios. Esse resultado indica que houve mudança, com melhora estatisticamente significativa $(\mathrm{p}=0,008)$. Em todos os 11 pacientes havia luxação no pré-operatório e, no pós-operatório, todos eles passaram a apresentar a articulação estável ou ausência de luxação, o que indica mudança estatisticamente significativa $(p=0,001)$. Entre os pacientes, nove apresentavam dor no pré-operatório e, no pós-operatório, apenas três, o que indica mudança estatisticamente significativa $(p=0,031)$. No pré-operatório, nove pacientes apresentaram limitação de atividade funcional e, no pós-operatório, em todos os 11 pacientes $(100,0 \%)$ havia ausência de limitação, o que indica mudança estatisticamente significativa $(\mathrm{p}=0,004)$. No préoperatório, seis pacientes apresentavam diminuição de força muscular e, no pós-operatório, apenas dois, porém, o resultado estatístico final não deve ser considerado como significativo $(\mathrm{p}=0,219)$. No pré-operatório, quatro pacientes apresentavam diminuição da mobilidade ativa quando comparada com a contralateral e, no pós-operatório, essa limitação não foi observada, mas esta diferença não é estatisticamente significativa $(\mathrm{p}=0,125)($ tabela 1$)$.

Análise da escala de Rowe - Nesta avaliação, a média préoperatória foi de 36,8 pontos e a média pós-operatória, de 81,8 pontos, havendo variação estatisticamente significativa. Todos os pacientes apresentaram aumento de pelo menos cinco pontos no escore geral e três pacientes tiveram escore máximo de 100 pontos. Foi encontrada variação significativa (p

TABELA 1

Resultados pela escala de Neer

\begin{tabular}{|c|c|c|c|c|c|c|c|c|c|c|c|c|}
\hline \multirow[t]{2}{*}{$\#$} & \multicolumn{2}{|c|}{ Luxações } & \multicolumn{2}{|c|}{ Dor } & \multicolumn{2}{|c|}{ Atividades } & \multicolumn{2}{|c|}{$\begin{array}{l}\text { Força } \\
\text { muscular }\end{array}$} & \multicolumn{2}{|c|}{ Mobilidade } & \multicolumn{2}{|c|}{ Resultado } \\
\hline & Pré & Pós & Pré & Pós & Pré & Pós & Pré & Pós & Pré & Pós & Pré & Pós \\
\hline 1 & $\mathrm{~S}$ & $\mathrm{~N}$ & $\mathrm{~S}$ & $\mathrm{~N}$ & $\mathrm{~S}$ & S & $\mathrm{S}$ & $\mathrm{S}$ & $\mathrm{N}$ & $\mathrm{S}$ & N.Satisf & Satisf \\
\hline 2 & $\mathrm{~S}$ & $\mathrm{~N}$ & $S$ & $\mathrm{~N}$ & $S$ & $S$ & $S$ & $S$ & $\mathrm{~N}$ & $\mathrm{~S}$ & N.Satisf & Satisf \\
\hline 3 & $\mathrm{~S}$ & $\mathrm{~N}$ & S & $\mathrm{S}$ & $S$ & S & $\mathrm{S}$ & $\mathrm{N}$ & S & $\mathrm{S}$ & N.Satisf & N.Satisf \\
\hline 4 & $\mathrm{~S}$ & $\mathrm{~N}$ & $S$ & $\mathrm{~S}$ & S & S & $\mathrm{N}$ & $\mathrm{N}$ & $\mathrm{S}$ & $\mathrm{S}$ & N.Satisf & N.Satisf \\
\hline 5 & $\mathrm{~S}$ & $\mathrm{~N}$ & S & $\mathrm{S}$ & $\mathrm{S}$ & S & $\mathrm{S}$ & S & S & $\mathrm{S}$ & N.Satisf & N.Satisf \\
\hline 6 & $\mathrm{~S}$ & $\mathrm{~N}$ & $S$ & $\mathrm{~N}$ & $\mathrm{~N}$ & $S$ & $\mathrm{~N}$ & $S$ & $\mathrm{~N}$ & $\mathrm{~S}$ & N.Satisf & Satisf \\
\hline 7 & $\mathrm{~S}$ & $\mathrm{~N}$ & $S$ & $\mathrm{~N}$ & $\mathrm{~N}$ & $\mathrm{~S}$ & $\mathrm{~N}$ & $\mathrm{~S}$ & $\mathrm{~N}$ & $\mathrm{~S}$ & N.Satisf & Satisf \\
\hline 8 & $\mathrm{~S}$ & $\mathrm{~N}$ & $S$ & $\mathrm{~N}$ & $\mathrm{~S}$ & $S$ & $\mathrm{~S}$ & $\mathrm{~S}$ & $\mathrm{~N}$ & $\mathrm{~S}$ & N.Satisf & Satisf \\
\hline 9 & $\mathrm{~S}$ & $\mathrm{~N}$ & $\mathrm{~N}$ & $\mathrm{~N}$ & $\mathrm{~S}$ & $\mathrm{~S}$ & $\mathrm{~N}$ & $\mathrm{~S}$ & $\mathrm{~N}$ & $\mathrm{~S}$ & N.Satisf & Satisf \\
\hline 10 & $S$ & $\mathrm{~N}$ & $S$ & $\mathrm{~N}$ & $S$ & $S$ & $S$ & $S$ & $S$ & $S$ & N.Satisf & Satisf \\
\hline 11 & $\mathrm{~S}$ & $\mathrm{~N}$ & $\mathrm{~N}$ & $\mathrm{~N}$ & $\mathrm{~S}$ & $S$ & $\mathrm{~N}$ & $\mathrm{~S}$ & $\mathrm{~N}$ & $\mathrm{~S}$ & N.Satisf & Satisf \\
\hline
\end{tabular}

Luxação = Nenhuma recidiva de luxação ou subluxação. Dor $=$ Presença de dor. Atividades = Atividades normais sem limitação. Força muscular $=$ Norma strength. Mobilidade $=$ Entre $10^{\circ}$ de extensão completa e $40^{\circ}$ de rotação comparado com o ombro contralateral. $\mathrm{N}=\mathrm{Não}, \mathrm{S}=\mathrm{Sim}$. Resultado: $\mathrm{N}$.Satisf $=$ Insatisfatório; Satisf = Satisfatório. 
$=0,002$ ) na pontuação de estabilidade do ombro, quando comparadas a avaliação pré e a pós-operatória. No pré-operatório, todos os pacientes tinham pontuação igual a zero e o aumento da pontuação no pós-operatório indica melhora significativa. Todos os pacientes apresentaram aumento de pelo menos 30 pontos na pontuação e, além disso, oito obtiveram pontuação máxima. Não foi encontrada variação estatisticamente significativa $(\mathrm{p}=0,102)$ em relação à mobilidade, comparadas a avaliação da média pré-operatória $(19,6)$ e a pós-operatória $(17,7)$. Dos 11 pacientes avaliados, cinco não alteraram a pontuação, um pacientes aumentou a pontuação em cinco pontos e cinco diminuíram a pontuação em cinco pontos. Houve variação estatisticamente significativa $(\mathrm{p}=0,011)$ em relação à função, quando se comparou a pontuação obtida na média pré-operatória $(19,1)$ com pós-operatória $(26,6)$. O aumento da pontuação indica melhora na avaliação pós-operatória. Oito pacientes apresentaram aumento de pelo menos cinco pontos na pontuação e três não alteraram a pontuação (tabela 2).

TABELA 2

Resultado da escala de Rowe

\begin{tabular}{|c|c|c|c|c|c|c|c|c|}
\hline \multirow[t]{2}{*}{ Paciente } & \multicolumn{2}{|c|}{ Estabilidade } & \multicolumn{2}{|c|}{ Mobilidade } & \multicolumn{2}{|c|}{ Função } & \multicolumn{2}{|c|}{ Total } \\
\hline & Pré & Pós & Pré & Pós & Pré & Pós & Pré & Pós \\
\hline 1 & 0 & 50 & 20 & 15 & 25 & 30 & 45 & 95 \\
\hline 2 & 0 & 50 & 20 & 15 & 10 & 30 & 30 & 95 \\
\hline 3 & 0 & 30 & 20 & 15 & 0 & 10 & 20 & 55 \\
\hline 4 & 0 & 50 & 20 & 20 & 25 & 25 & 45 & 95 \\
\hline 5 & 0 & 50 & 15 & 20 & 10 & 30 & 25 & 30 \\
\hline 6 & 0 & 30 & 20 & 15 & 25 & 25 & 45 & 70 \\
\hline 7 & 0 & 50 & 20 & 20 & 25 & 30 & 45 & 100 \\
\hline 8 & 0 & 50 & 20 & 20 & 30 & 30 & 30 & 100 \\
\hline 9 & 0 & 50 & 20 & 15 & 25 & 30 & 45 & 95 \\
\hline 10 & 0 & 30 & 20 & 20 & 10 & 25 & 30 & 75 \\
\hline 11 & 0 & 50 & 20 & 20 & 25 & 30 & 45 & 100 \\
\hline Média & 0 & 44,6 & 19,6 & 17,7 & 19,1 & 26,8 & 36,8 & 81,8 \\
\hline
\end{tabular}

Estabilidade $=$ Média dos resultados da estabilidade, pré $=$ pré-operatório e pós $=$ pós-operatório. Mobilidade $=$ Média dos resultados da mobilidade, pré $=$ pré-operatório e pós $=$ pós-operatório. Função $=$ Média dos resultados da função, pré $=$ préoperatório e pós $=$ pós-operatório. Total $=$ Resultado da média da escala pré $=$ préoperatório e pós = pós-operatório.

Avaliação da escala da UCLA - O resultado médio no préoperatório foi de 23,45 pontos e, no pós-operatório, de 31,18 pontos, observando-se variação estatisticamente significativa $(\mathrm{p}=0,005)$. $\mathrm{O}$ aumento na pontuação indica melhora significante na avaliação pós-operatória, na qual nove pacientes apresentaram aumento de pelo menos um ponto no escore geral e dois tiveram a pontuação máxima (35 pontos) (tabela 3). Não foi encontrada variação estatisticamente significativa $(\mathrm{p}=$ $0,168)$ na média da dor pré-operatória de 6,73 pontos para o pós-operatório de 7,45 pontos. Dos 11 pacientes operados, dois $(18,2 \%)$ não alteraram a pontuação, sete $(63,2 \%)$ aumentaram a pontuação e dois diminuíram a pontuação. Quanto à função, foi encontrada variação estatisticamente significativa $(\mathrm{p}=0,020)$, quando se comparou a média no pré-operatório (7,45 pontos) com a no pós-operatório (9,27 pontos). $\mathrm{O}$ aumento na pontuação indica que houve melhora significante na avaliação pós-operatória: oito pacientes apresentaram aumento de pelo menos dois pontos no escore, dois não alteraram a pontuação e um a diminuiu em dois pontos.

TABELA 3

Resultados da escala de Neer

\begin{tabular}{lcc}
\hline & Pré-op & Pós-op \\
\hline Dor & 6,73 & 7,45 \\
Função & 7,45 & 9,27 \\
Elevação & 4,73 & 4,82 \\
Força & 4,55 & 4,64 \\
Satisfação & 0 & 5 \\
Pontuação final & 23,45 & 31,18
\end{tabular}

Dor $=$ Média dos resultados da dor, pré $=$ pré-operatório e pós $=$ pós-operatório. Função $=$ Média dos resultados da função, pré $=$ pré-operatório e pós = pós-operatório Elevação = Média dos resultados da elevação ativa, pré $=$ pré-operatório e pós = pósoperatório. Elevação = Média dos resultados da força da elevação ativa, pré = préoperatório e pós = pós-operatório. Satisfação = Média dos resultados quanto a satis fação e melhora do paciente, pré = pré-operatório e pós = pós-operatório.

$\mathrm{O}$ resultado da avaliação pré-operatória foi ruim em dois pacientes, regular em oito e bom em um. No pós-operatório, o resultado encontrado foi regular em dois pacientes $(18,2 \%)$, bom em sete $(63,6 \%)$ e excelente em dois $(18,2 \%)$.

Avaliação pela escala de ASES - Foi aplicada a escala de ASES na avaliação pós-operatória dos 11 pacientes operados. A pontuação variou entre 10 e 98,3 pontos, com média de 77,2 pontos $(\mathrm{DP}=27,9)$ e mediana de 89,7 . Em relação à escala visual da dor: a pontuação variou entre 0 e 7 pontos, com média de 1,7 pontos $(\mathrm{DP}=2,2)$ e mediana de 1 ponto.

\section{DISCUSSÃO}

As cirurgias abertas convencionais para tratamento da instabilidade multidirecional do ombro apresentam resultados favoráveis, mesmo quando avaliados a longo prazo. Realizamos uma revisão dos casos operados em nosso serviço e os comparamos com a literatura pertinente, em relação à via aberta e à artroscópica. Neer et al descreveram uma técnica aberta 
de capsulorrafia em que é feita a desinserção umeral da cápsula articular, que é dividida em duas bandas; a inferior é avançada para uma posição mais superior no úmero, o que diminui o volume do recesso axilar capsular ${ }^{(2)}$. Com essa técnica, os autores observaram $97 \%$ de resultados satisfatórios. A única recidiva encontrada estava relacionada com lesão de Bankart ${ }^{(2)}$. A capsulorrafia realizada por desinserção da cápsula na glenóide, descrita por Alcheck et al, associada a reparo da lesão do lábio da glenóide, apresenta $10 \%$ de recidivas em relação à instabilidade ${ }^{(8)}$. Nos estudos de Neer et al e de Alcheck et al, há relato de redução da amplitude de movimento quanto à rotação externa e, em certos casos, evolução para artrose. Observamos redução da rotação externa. É possível que, a médio ou longo prazo, haja evolução para artrose.

A redução do volume capsular por via artroscópica foi inicialmente descrita através da técnica por sutura transglenoidal, por sutura da cápsula com fios e por capsulorrafia térmica, associada ou não a outros procedimentos. Duncan et al propuseram uma técnica de incisão da cápsula articular avançando sua porção inferior para uma posição mais superior, fixando o conjunto por sutura transglenoidal ${ }^{(9)}$. Complementaram esse procedimento com a sutura do espaço rotador. Em pacientes com lesão de Bankart, obtiveram 100\% de estabilidade. Em pacientes sem lesão de Bankart, 95\% deles permaneceram estáveis ${ }^{(9)}$. Treacy et al encontraram resultados semelhantes no tratamento da instabilidade multidirecional por capsulorrafia e sutura transglenoidal ${ }^{(10)}$. Esses resultados indicam a importância da abordagem artroscópica do volume capsular com resultados próximos aos encontrados na via convencional e aos observados no nosso estudo. Apesar desses resultados favoráveis, na avaliação a longo prazo da sutura transglenoidal, os pacientes com lesão do lábio da glenóide não parecem apresentar resultados satisfatórios. Walch et al encontraram recidiva em $49 \%$ dos casos ${ }^{(11)}$ e Green et al, em $42 \%$. Nos casos de recidiva, Green et al identificaram insuficiência do ligamento glenoumeral inferior ${ }^{(7)}$. Os fios de sutura fixados na fáscia posterior em um tecido edemaciado podem influenciar nos resultados desfavoráveis. A capsulorrafia térmica permite a abordagem de toda a extensão da cápsula articular, não sendo necessária a perfuração de um canal ósseo na glenóide, cuja complicação associada pode estar relacionada a lesão vasculonervosa. A sutura transglenóide foi abandonada e substituída por outros procedimentos.

Snyder et al descreveram uma técnica de redução de volume articular por múltiplas suturas diretas na cápsula, em pacientes sem lesão do lábio da glenóide, e, em seu seguimento, todos os pacientes permaneceram estáveis ${ }^{(12)}$. Os seus maus resultados estão todos relacionados à dor. Não foram descritos casos de necrose capsular após a sutura por fios, mas, como em nosso estudo, não houve reavaliação artroscópica de todos os pacientes tratados que permitisse confirmar a ausência dessa lesão.

Procedimento por via artroscópica com capsulorrafia por uso de energia térmica, com utilização de radiofrequiência ou laser, é técnica mais simples e rápida. Noonan et al operaram pacientes com e sem lesão do lábio da glenóide por capsulorrafia térmica por meio da radiofrequiência monopolar, mas não suturaram o espaço rotador. Encontraram $86,3 \%$ de pacientes estáveis na instabilidade multidirecional ${ }^{(13)}$. Enad et al realizaram a capsulorrafia com o uso de laser, não suturaram espaço rotador e obtiveram $83 \%$ de ombros estáveis. Esses autores associaram a taxa de recidivas ao não fechamento do espaço rotador $^{(14)}$. Lyons et al realizaram capsulorrafia a laser associada à sutura do espaço rotador apenas nos casos em que o espaço não se fechava com o tratamento térmico e obtiveram $96 \%$ de ombros estáveis ${ }^{(15)}$. Considerando esses resultados, acreditamos que a abordagem do espaço rotador é um procedimento necessário para complementação da capsulorrafia, e isso foi feito em todos os casos do nosso estudo.

Observamos que houve melhora nas três escalas quando o pré e o pós-operatório foram comparados. Considerando os resultados encontrados na escala de Neer, $72,7 \%$ dos pacientes permaneceram satisfeitos e os resultados insatisfatórios estão relacionados com presença de dor. Duncan et al, com a sutura transglenoidal artroscópica, associada à sutura do espaço rotador, obtiveram $100 \%$ de resultados satisfatórios ${ }^{(9)}$. Wichman et al encontraram $79 \%$ de resultados satisfatórios com a sutura mecânica da cápsula ${ }^{(16)}$. Lyons et al realizaram capsulorrafia a laser associada à sutura do espaço rotador e encontraram $88 \%$ de resultados satisfatórios ${ }^{(15)}$.

Considerando-se a escala da UCLA, obtivemos melhora estatisticamente significativa com resultado favorável - de bom a excelente - em $81,8 \%$ dos casos. Favorito et al, realizando exclusivamente a capsulorrafia a laser, encontraram resultado favorável entre bom e excelente em 81,5 dos $\operatorname{casos}^{(17)}$.

Na avaliação pós-operatória pela escala ASES, observamos pontuação de 77,2. Essa média é próxima à encontrada por Enad et al com capsulorrafia e sutura do espaço, com a qual obteve resultado de 84,2 pontos $^{(14)}$. Noonan et al, com a capsulorrafia térmica por radiofrequiência, sem sutura do espaço rotador, obtiveram 83,9 pontos em pacientes sem lesão do lábio da glenóide ${ }^{(13)}$.

A dor nas atividades foi a principal causa de nossos maus resultados. Contudo, a análise da dor, usando-se a escala de 
Neer, indicou melhora da pontuação. Utilizando a escala da UCLA, não foi observada melhora estatisticamente significativa em relação à dor, mas tendência para melhora. No estudo desenvolvido por Wichman et al, todos os maus resultados também estavam associados à dor ${ }^{(16)}$.

Este estudo não considerou um grupo controle, no qual o tratamento artroscópico fosse feito exclusivamente pelo fechamento do espaço rotador. Assim, não poderemos afirmar se esse procedimento realizado por vários pontos seria suficiente para o tratamento da instabilidade multidirecional.

Houve melhora significativa nas escalas de Neer e UCLA quanto à função. Houve melhora quanto à mobilidade, segundo os critérios de Neer, mas essa melhora não foi significativa nas escalas de UCLA e Rowe. Existe diminuição no movimento de rotação externo, no teste de mobilidade passiva no pósoperatório, mas esse movimento não causou apreensão. Essa situação permitiu a realização de movimentos de rotação antes evitados. Por isso, consideramos que houve melhora nesse critério.

\section{CONCLUSÃO}

O procedimento artroscópico analisado confere estabilidade e melhora funcional.

\section{REFERÊNCIAS}

1. Speer KP, Deng X, Borrero S, Torzilli PA, Altchek DA, Warren RF. Biomechanical evaluation of a simulated Bankart lesion. J Bone Joint Surg Am. 1994;76(12):1819-26.

2. Neer CS, Foster CR. Inferior capsular shift for involuntary inferior and multidirectional instability of the shoulder: a preliminary report. 1980. J Bone Joint Surg Am. 2001;83-A(10):1586.

3. Nelson BJ, Arciero RA. Arthroscopic management of glenohumeral instability. Am J Sports Med. 2000;28(4):602-14.
4. Ellman H, Kay SP. Arthroscopic subacromial decompression for chronic impingement. Two- to five-year results. J Bone Joint Surg Br. 1991; 73(3):395-8.

5. Rowe CR, Patel D, Southmayd WW. The Bankart procedure: a long-term end-result study. J Bone Joint Surg Am. 1978;60(1):1-16.

6. King GJ, Richards RR, Zuckerman JD, Blasier R, Dillman C, Friedman RJ, et al. A standardized method for assessment of elbow function. Research Committee, American Shoulder and Elbow Surgeons. J Shoulder Elbow Surg. 1999;8(4):351-4.

7. Green MR, Christensen KP. Arthroscopic Bankart procedure: two- to fiveyear followup with clinical correlation to severity of glenoid labral lesion. Am J Sports Med. 1995;23(3):276-81.

8. Altchek DW, Warren RF, Skyhar MJ, Ortiz G. T-plasty modification of the Bankart procedure for multidirectional instability of the anterior and inferior types. J Bone Joint Surg Am. 1991;73(1):105-12.

9. Duncan R, Savoie FH. Arthroscopic inferior capsular shift for multidirectional instability of the shoulder: a preliminary report. Arthroscopy. 1993;9(1):24-7.

10. Treacy SH, Field LD, Savoie FH. Rotator interval capsule closure: an arthroscopic technique. Arthroscopy. 1997;13(1):103-6.

11. Walch G, Boileau P, Levigne C, Mandrino A, Neyret P, Donell S. Arthroscopic stabilization for recurrent anterior shoulder dislocation: results of 59 cases. Arthroscopy. 1995;11(2):173-9.

12. Snyder SJ, Strafford BB. Arthroscopic management of instability of the shoulder. Orthopedics. 1993;16(9):993-1002.

13. Noonan TJ, Tokish JM, Briggs KK, Hawkins RJ. Laser-assisted thermal capsulorrhaphy. Arthroscopy. 2003;19(8):815-9.

14. Enad JG, Kharrazi FD, ElAttrache NS, Yocum LA. Electrothermal capsulorrhaphy in glenohumeral instability without Bankart tear. Arthroscopy. 2003;19(7):740-5.

15. Lyons TR, Griffith PL, Savoie FH, Field LD. Laser-assisted capsulorrhaphy for multidirectional instability of the shoulder. Arthroscopy. 2001;17(1):25-30.

16. Wichman MT, Snyder SJ. Operative Techniques in sports medicine Sports Med 5:238-243, 1997. Wichman MT, Snyder SJ. Arthroscopic capsular plication for multidirectional instability of the shoulder. Oper Tech Sports Med. 1997;5(4):238-43.

17. Favorito PJ, Langenderfer MA, Colosimo AJ, Heidt RS, Carlonas RL. Arthroscopic laser-assisted capsular shift in the treatment of patients with multidirectional shoulder instability. Am J Sports Med. 2002;30(3):322-8. 